\title{
Autocuidado del adulto mayor con incontinencia urinaria en dos municipios del Estado de Hidalgo
}

\section{Self-care of the elderly with urinary incontinence in two municipalities of the State of Hidalgo}

\author{
Claudia A. Trejo-García ${ }^{a}$, Gabriela Maldonado-Muñiz ${ }^{b}$, Benjamín López-Nolasco ${ }^{c}$, Daniel \\ Cruz-Mendoza ${ }^{d}$, Lizbeth Trejo-Martinez ${ }^{e}$
}

\begin{abstract}
:
Urinary incontinence (UI) is a prevalent syndrome that usually occurs in old age. It is caused by several factors that happen in the elder adult stage. However, it should not be considered as a normal state since it is not a physiological result of aging process. Objective: To evaluate the level of self-care in the elder adult who suffer urinary incontinence, in two communities of the State of Hidalgo. Methodology: This is a quantitative, descriptive, cross-sectional study. Non-probabilistic sample due to availability conformed by 162 elder adults from the communities of Tasquillo and Tula de Allende Hidalgo. Once the protocol was approved by the Ethics Committee, the data was collected with prior informed consent. The instruments ICIQ-SF (International Consultation on Incontinence Questionnaire - Short Form) with a reliability of 0.87 by Cronbach's coefficient and the Self-care instrument for the elderly with urinary incontinence validated ex-profeso, with a reliability by Kuder-Richardson coefficient of 0.79 were used. Results: The Elder adult characterization of the basic conditioning factors reported ages that ranged from 60 to 95 years with a predominance in the group of 72-77 years, the predominant gender was female with 57\%, the marital status highlighted the category widower with $36 \%$, in relation to schooling highlights $43 \%$ with secondary level studies, $35 \%$ of the study population professed the Catholic religion. Regarding the types of urinary incontinence, $39.5 \%$ were found with stress urinary incontinence, $88 \%$ showed inadequate self-care.
\end{abstract}

\section{Keywords:}

Elderly, self - care, urinary incontinence

\section{Resumen:}

La incontinencia urinaria (IU) es un síndrome que se presenta comúnmente en la etapa de la vejez, la causa se debe a diversos factores que se presentan en la etapa de adulto mayor, sin embargo, no debe considerarse como un estado normal, ya que no es un resultado fisiológico del proceso de envejecimiento. Objetivo: Evaluar el nivel de autocuidado del adulto mayor con incontinencia urinaria en dos municipios del Estado de Hidalgo. Metodología: estudio cuantitativo, descriptivo, de corte transversal, muestra no probabilística por disponibilidad constituida por 162 adultos mayores de los municipios de Tasquillo y Tula de Allende Hidalgo. Una vez aprobado el protocolo por el Comité de ética, se realizó la recolección de datos previo consentimiento informado, se utilizaron los instrumentos: ICIQ-SF (International Consultation on Incontinence Questionnaire - Short Form) con una confiabilidad de 0.87 por coeficiente de Cronbach y el instrumento autocuidado del adulto mayor con incontinencia urinaria validado ex-profeso, con una confiabilidad por coeficiente de Kuder-Richardson de 0.79. Resultados: La caracterización de los Factores condicionantes básicos de los adultos mayores reporto edades que oscilaron entre los 60 a 95 años, predominando el grupo de 72-77 años, el género predominante fue el femenino con un 57\%, el estado civil destacó la categoría de viudo con un 36\%, en relación a la escolaridad destaca $43 \%$ con estudios

\footnotetext{
a Autor de Correspondencia, Universidad Autónoma del Estado de Hidalgo, Escuela Superior de Tlahuelilpan, https://orcid.org/0000-00032205-7879, Email: ctrejo@uaeh.edu.mx

b Universidad Autónoma del Estado de Hidalgo, Escuela Superior de Tlahuelilpan, https://orcid.org/0000-0002-4967-1812, Email: gmaldonado@uaeh.edu.mx

Universidad Autónoma del Estado de Hidalgo, Escuela Superior de Tlahuelilpan, https://orcid.org/0000-0003-4566-214X, Email: benjamin_lopez8596@uaeh.edu.mx

d Universidad Autónoma del Estado de Hidalgo, Escuela Superior de Tlahuelilpan, https://orcid.org/0000-0003-1533-4970, Email: cr77938@uaeh.edu.mx

Universidad Autónoma del Estado de Hidalgo, Escuela Superior de Tlahuelilpan, https://orcid.org/0000-0002-8935-6104, Email: tr382759@uaeh.edu.mx
} 
de nivel secundaria, 35\% de la población de estudio profesaba la religión católica. Respecto a los tipos de incontinencia urinaria se encontró $39.5 \%$ con incontinencia urinaria de esfuerzo, $88 \%$ mostró un autocuidado inadecuado.

\section{Palabras Clave:}

Adulto mayor, autocuidado, incontinencia

\section{Introducción}

México es un país que se encuentra en constante cambio, así mismo la población se encuentra inmersa en un proceso dinámico que lentamente la ha llevado a una transición demográfica que la convierte en una población envejecida. [1]

Al incrementar la esperanza de vida en la población mexicana, se enfrenta un gran reto social, el envejecimiento de la población, los mayores de 65 años se van incrementando gradualmente, lo que con lleva a comprometer la capacidad de autocuidado del adulto mayor. El envejecimiento en términos generales trae consigo una serie de cambios físicos, fisiológicos, los cuales se ven influenciados por consumo de fármacos, modificaciones en la composición corporal, disminución en la función renal, incontinencia urinaria entre otras.

La incontinencia Urinaria (IU) es la pérdida involuntaria de orina que causa molestias a la persona, ya que se presenta en momentos o lugares de manera inesperada [2]. Es un problema de salud importante en la población de adultos mayores, pero también incluye a la familia y/o cuidadores, la importancia de esta alteración no se expresa en términos de gravedad, pero si en la alteración de la calidad de vida del adulto mayor.

La International Continence Society define la IU como cualquier queja de salida de orina que provoque molestias al paciente, se presenta cuando la uretra no puede aguantar la presión que ejerce la orina contenida en la vejiga. Cualquier pérdida involuntaria de orina ocurrida en al menos seis ocasiones durante el año o cualquier historia evidente de goteo inducido por estrés o urgencia, es considerada incontinencia urinaria. [3].

En términos generales la $\mathrm{IU}$ es el resultado de la disfuncionalidad de los mecanismos de continencia, alterando el llenado vesical, almacenamiento de orina y su vaciamiento, provocando la pérdida involuntaria de la misma.

La IU en el adulto mayor se considera un síndrome de etiología múltiple, ya que, a diferencia del adulto joven, donde los factores de tipo urológico juegan el papel principal en la fisiopatología, etiología y manifestaciones clínicas asociadas a la incontinencia, en el adulto mayor se suman los cambios propios del envejecimiento, polifarmacia, polipatología, cuestiones culturales, económicas y sociales que intervienen de manera directa o indirecta en la presencia de la IU. [4].
Esta condición tiene una repercusión sobre el entorno social y familiar del adulto mayor, ya que la mayoría de los adultos mayores incontinentes tienden a ser institucionalizados, además de demostrar un deterioro en la percepción de su calidad de vida y un aumento en los trastornos emocionales y aislamiento social.

La IU es una alteración de las necesidades básicas de individuo, es un diagnóstico de enfermería que involucra problemas de higiene y sociales que influyen en el entorno del adulto mayor y en su relación con los demás.

Cuadro 1. Clasificación de la incontinencia urinaria de etiología urológica de acuerdo al mecanismo fisiopatológico de origen y características clínicas sobresalientes

\begin{tabular}{|c|c|c|}
\hline $\begin{array}{l}\text { Tipo de } \\
\text { Incontinencia }\end{array}$ & $\begin{array}{l}\text { Mecanismo } \\
\text { Fisiopatológico }\end{array}$ & $\begin{array}{l}\text { Características } \\
\text { Clínicas Sobresalientes }\end{array}$ \\
\hline De esfuerzo & $\begin{array}{l}\text { El esfínter uretral } \\
\text { deficiente con } \\
\text { disminución } \\
\text { clínicamente } \\
\text { significativa de la } \\
\text { presión máxima de } \\
\text { cierre uretral con la } \\
\text { presencia de } \\
\text { cualquier factor que } \\
\text { aumente la presión } \\
\text { de la cavidad } \\
\text { abdominal. }\end{array}$ & $\begin{array}{l}\text { Presencia de fugas } \\
\text { urinarias asociadas a la } \\
\text { presencia de tos, } \\
\text { estornudos, o bien } \\
\text { actividad f }\end{array}$ \\
\hline Con urgencia: & $\begin{array}{l}\text { Disfunción e } \\
\text { inestabilidad del } \\
\text { detrusor por } \\
\text { alteración neuronal } \\
\text { periférica o central } \\
\text { (lesión medular } \\
\text { sacra, secuelas de } \\
\text { EVC, Parkinson, } \\
\text { Alzheimer, etc.) } \\
\text { Cursa con } \\
\text { contracciones } \\
\text { vesicales } \\
\text { involuntarias. }\end{array}$ & $\begin{array}{l}\text { Polaquiuria, tenesmo } \\
\text { vesical, enuresis } \\
\text { nocturna, deseo } \\
\text { imperioso de orinar, esta } \\
\text { signo-sintomatología se } \\
\text { exacerba generalmente } \\
\text { con la presencia de } \\
\text { procesos infecciosos } \\
\text { urinarios. También es } \\
\text { común que los síntomas } \\
\text { se exacerben con la } \\
\text { ingestión de irritantes } \\
\text { vesicales. }\end{array}$ \\
\hline $\begin{array}{l}\text { Por } \\
\text { Rebozamiento }\end{array}$ & $\begin{array}{l}\text { Se caracteriza por la } \\
\text { obstrucción } \\
\text { mecánica de la } \\
\text { uretra o } \\
\text { imposibilidad para la } \\
\text { contracción del } \\
\text { retrusor evitando la } \\
\text { micción normal. } \\
\text { Cuando el volumen } \\
\text { urinario rebasa la } \\
\text { presión ejercida por }\end{array}$ & $\begin{array}{l}\text { El chorro de la orina se } \\
\text { emite de forma } \\
\text { intermitente, disminuido } \\
\text { en su calibre, } \\
\text { acompañándose de pujo } \\
\text { vesical, polaquiuria y } \\
\text { goteo miccional terminal. } \\
\text { En presencia de gran } \\
\text { retención urinaria se } \\
\text { presenta globo vesical. } \\
\text { En ocasiones, el }\end{array}$ \\
\hline
\end{tabular}




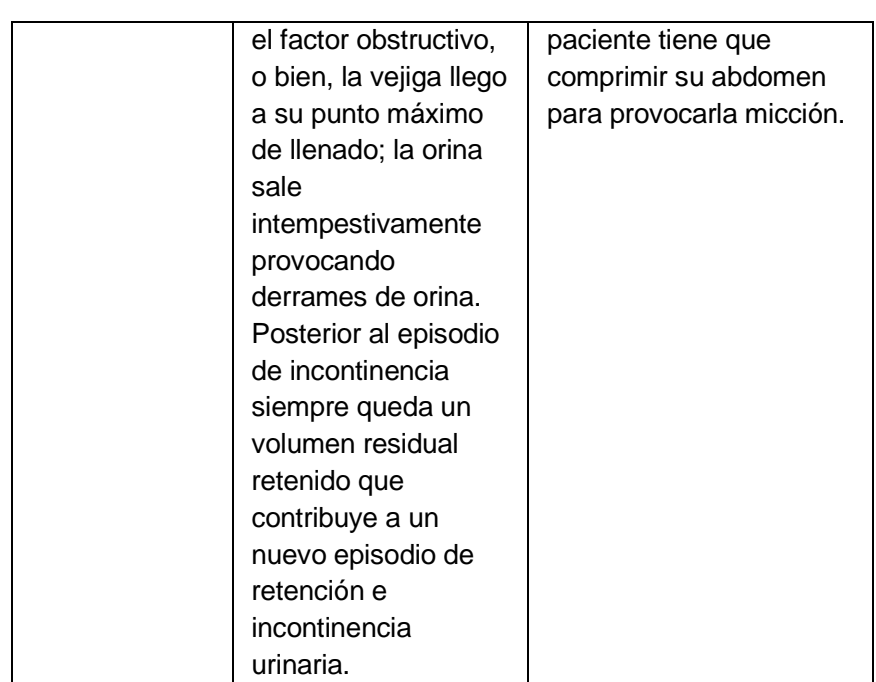

Fuente: Centro Nacional de Programas Preventivos y control de Enfermedades. Incontinencia Urinaria en la Persona Adulta Mayor. Guía de consulta para el médico de primer nivel. (2010)

Un punto importante que contribuye a la mejora del adulto mayor es el autocuidado ya que involucra a la persona mayor como actor competente capaz de tomar decisiones, controlar su propia vida y asegurarse la posibilidad de gozar de un buen estado de salud. En este sentido, el autocuidado le permitirá alcanzar una mejor calidad de vida, a través del fortalecimiento del potencial de autonomía y de la responsabilidad de sí mismo, indispensables para el logro de su envejecimiento pleno y saludable.

En el caso del adulto mayor con IU, esta perspectiva le favorece a tomar conciencia de su propia condición, mediante la mejora de sus conocimientos y habilidades, orientados hacia un cambio de comportamiento positivo, que le permita reducir el riesgo de complicaciones.

El autocuidado convierte a la persona de edad avanzada en motor de su propio bienestar, y con el apoyo familiar puede encontrar la solución a la mayoría de sus problemas.

\section{Planteamiento del problema}

La Organización Mundial de la Salud (OMS), considera que el número de personas mayores va en aumento, tiene proyecciones que entre 2015 y 2015 se duplicará la cantidad de adultos. [5]

Varios de los problemas de salud que padecen los adultos mayores son atribuibles a la edad; sin embargo muchos de ellos se deben a los inadecuados hábitos adquiridos durante las etapas previas de su vida y que se potencializan por la edad, entre ellas la IU.

La IU además de ser considerada un problema médico, tiene repercusiones sociales importantes; no es un proceso que ponga en riesgo la vida de los adultos mayores, sin embargo, deteriora de manera significativa la calidad de vida de las personas, limitando con ello su autonomía y reduciendo su autoestima, también puede conducir a un declive físico, funcional y emocional con consecuencias funcionales, psicosociales e incluso económicas, además, la urgencia por orinar es causa del incremento de caídas y fracturas en varias ocasiones. No obstante, esta situación pasa desapercibida, ya que la mayoría de las personas, lo consideran como problemas naturales generados por la edad avanzada o bien por prejuicios sociales tienden a ocultarla, impidiendo con ello los tratamientos oportunamente, aunado a la falta de autocuidado de los adultos mayores.

Por lo que surgió la siguiente pregunta de investigación: ¿Cuál es el nivel de autocuidado del adulto mayor con incontinencia urinaria en dos municipios del Estado de Hidalgo?

\section{Conocimiento Previo}

Existen más de 200 millones de individuos en todo el mundo que tienen problemas asociados a la incontinencia urinaria. Se considera como la segunda causa de internamiento en casas de la tercera edad. Aproximadamente, $30 \%$ de los adultos mayores de 60 años y $50 \%$ de los adultos institucionalizados, presentan algún grado de incontinencia urinaria.

Un estudio reciente en cuatro países europeos (Francia, Alemania, Reino Unido y España) sobre las respuestas de una encuesta por correo a 17, 080 mujeres de 18 años y más, reportó que un $35 \%$ refirieron pérdidas involuntarias de orina en los últimos 30 días. La tasa de prevalencia de IU fue similar en todos los países (41 a 44\%), excepto en España que fue menor (23\%).

En ambos estudios se demuestra claramente que la prevalencia de IU aumenta con la edad. [2]

\section{Metodología de diseño o estudio}

Estudio cuantitativo, descriptivo, de corte transversal, muestra no probabilística por disponibilidad constituida por 162 adultos mayores de los municipios de Tasquillo y Tula de Allende Hidalgo. Una vez aprobado el protocolo por el Comité de ética, se realizó la recolección de datos previo consentimiento informado, se utilizaron los instrumentos: ICIQ-SF (International Consultation on Incontinence Questionnaire - Short Form) integrado por tres ítems con los que se identifica la presencia de incontinencia urinaria y una cuarta para conocer el tipo de incontinencia urinaria, con una confiabilidad de 0.87 por coeficiente de Cronbach, y el instrumento autocuidado del adulto mayor con incontinencia urinaria, el cual está constituido por 21 items.

Cada pregunta tiene la posibilidad de ser valorada presentando las opciones de respuesta SI con valor de 1 y NO con valor de 0 , siendo mayor de 11 respuestas 
negativas se considera como un autocuidado inadecuado. Se realiza validación ex - profeso en población mexicana, con una población para prueba piloto conformada por 25 adultos mayores, obteniendo una confiabilidad por coeficiente de Kuder-Richardson de 0.79 .

\section{Aplicación}

En este estudio se toma como referente teórico la teoría del autocuidado, en la donde Orem describe el autocuidado haciendo un análisis semántico de la palabra, la cual separa en "AUTO" y que define cono el individuo integral, incluyendo no solo sus necesidades físicas, sino también las psicológicas y espirituales"; y "CUIDADO", que define como "la totalidad de las actividades que un individuo inicia para mantener la vida y desarrollarse de una forma que sea normal para él".

Devela el origen del autocuidado como "un comportamiento aprendido", y las actividades de autocuidado como acciones que se aprenden e internalizan tras la interacción e influencias del medio y la vida cultural del grupo al cual pertenece al individuo. [6]

\section{Resultados}

La caracterización de los Factores condicionantes básicos de los adultos mayores reporto edades que oscilaron entre los 60 a 95 años, predominando el grupo de 72-77 años, el género predominante fue el femenino con un $57 \%(n=92)$, el masculino con un $43 \%(n=70)$. En el estado civil destacó la categoría de viudo con un $36 \%$ $(n=58)$, seguido de casado con $30 \%(n=49)$ soltero $18 \%$ $(n=30)$ y divorciado $15 \% \quad(n=25)$. En relación a la escolaridad destaca $43 \%(n=69)$ con estudios de nivel secundaria, $38 \% \quad(n=62) \quad$ primaria, $18 \% \quad(n=29)$ preparatoria y solo el $1 \%(n=2)$ cuentas con estudios universitarios. $35 \%$ de la población son creyentes, 34\% $(\mathrm{n}=28)$ profesan la religión cristiana.

El 53\% $(n=86)$ de la población pertenece a Tasquillo y el $47 \%(n=76)$ pertenece a la comunidad de Tula, ambos municipios del estado de Hidalgo.

Una vez evaluada la presencia de IU en la población de estudio se identificó que $40 \% \quad(n=64)$ presentan incontinencia urinaria mixta, seguida con $36 \%(n=59)$ con incontinencia urinaria de esfuerzo, $14 \% \quad(n=22)$ incontinencia urinaria de urgencia, solo el $10.5 \%$ ( $n=(17)$ presenta incontinencia funcional.

En cuanto a la variable autocuidado del adulto mayor con incontinencia se encontró que el $88 \%(n=143)$ presenta un nivel de autocuidado inadecuado.
De acuerdo a los resultados obtenidos, el autocuidado que realizan los adultos mayores se encontró que el $12 \%$ $(n=19)$ de la población de dos municipios del Estado de Hidalgo presenta un autocuidado adecuado y el $88 \%$ $(n=143)$ es inadecuado, coincidiendo con lo mencionado por la teórica Dorothea E. Orem, quien en su Teoría General del Déficit de autocuidado, menciona que los adultos mayores tienen limitaciones de sus acciones relacionadas con su salud o con el cuidado de salud. [7] La incontinencia urinaria contiene diversas clasificaciones, en el trabajo de investigación realizado con ayuda del instrumento ICIQ, se puede identificar los tipos de incontinencia urinaria de: esfuerzo, urgencia, mixta y funcional. Dentro del presente trabajo de investigación se obtuvo una mayor significancia en la Incontinencia urinaria mixta $(39.5 \%, n=64)$, contrario a lo expuesto por Misericordia G. H. en su trabajo denominado "Tipos de incontinencia urinaria en personas mayores de 65 años institucionalizadas: tratamiento y cuidados" publicado en el año 2013 en España, donde se tuvo un mayor porcentaje en cuanto a la incontinencia urinaria de esfuerzo (54\%), esto debido a que los adultos mayores pierden parte de su fuerza física, por lo que, un esfuerzo mayor o brusco ocasiona en el adulto mayor una perdida involuntaria de orina.

\section{Conclusiones}

- En base a los resultados obtenidos en dos municipios del Estado de Hidalgo, el autocuidado del adulto mayor con incontinencia urinaria es inadecuado.

- Considerando que la IU es un diagnóstico de enfermería el cual puede atenderse mediante diversas intervenciones, se puede lograr incrementar el nivel de autocuidado del adulto mayor considerando la Teoría de Sistemas, específicamente mediante el apoyo educativo, logrando con ello que el adulto mayor adquiera conocimientos, desarrolle habilidades, mejorando con ello su calidad de vida.

\section{Referencias}

[1] CENAPRECE, «Centro Nacional de Programas Preventivos y Control de Enfermedades,» 26 abril 2018. [En línea]. Available: www.cenaprece.salud.gob.mx/programas/interior/adulto/envejecimie nto/portada_envejecimiento.

[2] CENETEC, «Catalogo maestro - guías de práctica clinica,» 10 septiembre 2018. [En línea]. Available: http://www.cenetecdifusion.com/CMGPC/GPC-IMSS-190-18/ER.pdf. [Último acceso: 2020].

[3] Instituto Nacional de Geriatría, Boletín Instituto Nacional de Geriatría, vol. 2, nº 6, p. 12, Julio 2019.

[4] Secretaria de Salud, «Centro Nacional de Programas Preventivos y Control de Enfermedades,» 2010. [En línea]. Available:

\section{Discusión}


Publicación semestral, XIKUA Boletín Científico de la Escuela Superior de Tlahuelilpan, Vol. 9, No. 18 (2021) 1-5

http://www.cenaprece.salud.gob.mx/programas/interior/adulto/desca rgas/pdf/Guia_primer_nivel_incontinencia_urinaria.pdf.

[5] Organización Mundial de la Salud, «Organización Mundial de la Salud,» 2015. [En línea]. Available:

https://www.who.int/ageing/about/facts/es/. [Último acceso: 0410 2020].

[6] N. D. C. Gálvez Díaz, «Repositorio.uss,» 01 julio 2017. [En línea]. Available:

http://repositorio.uss.edu.pe/bitstream/handle/uss/3049/MENDO-

FLORES.pdf?sequence=5\&isAllowed=y. [Ültimo acceso: 0409

2020].

[7] V. Berbiglia y B. Banfield, «Teoría del déficit de autocuidado,» de Modelos y teorías de enfermería, España, Elsevier, 2015, pp. 232 . 248. 